\title{
IMPACTO DE GESTIÓN DE LA RESPONSABILIDAD SOCIAL UNIVERSITARIA DEL INSTITUTO DE POSTGRADO DE LA UNIVERSIDAD TÉCNICA DE MANABÍ
}

\section{IMPACT OF THE MANAGEMENT OF UNIVERSITY SOCIAL RESPONSIBILITY OF THE POSTGRADUATE INSTITUTE OF THE TECHNICAL UNIVERSITY OF MANABÍ}

\author{
Zambrano-Mendoza Maika Sofia ${ }^{1 *}$; Vivas-Vivas Esperanza² \\ ${ }^{1}$ Maestrante Programa de Posgrado Gerencia de la Calidad de Innovación, \\ Universidad Técnica de Manabí, UTM. Portoviejo, Ecuador. \\ ${ }^{2}$ Docente de la Universidad Técnica de Manabí, UTM. Portoviejo, Ecuador.
}

*Correo: sofia.ecu@hotmail.com

\begin{abstract}
Resumen
El presente trabajo analiza el impacto de gestión de la responsabilidad social universitaria, tomando en cuenta que en los últimos años este tema ha ganado terreno en el ámbito académico, a nivel local, nacional e internacional. Por ello está enfocado en el instituto de posgrado de la Universidad Técnica de Manabí, como un espacio de reflexión a la acción social que éste debe generar entre sus estudiantes, donde el profesional disponga de la capacidad para aportar con soluciones viables a problemas de carácter social basados en la realidad actual.
\end{abstract}

Palabras claves: Responsabilidad social universitaria, estudiantes, docentes, ética, gestión, impacto.

\begin{abstract}
This paper analyzes the impact of university social responsibility management, taking into account that in recent years this issue has gained ground in the academic field, at the local, national and international levels. For this reason, it is focused on the postgraduate institute of the Technical University of Manabí, as a space for reflection on the social action that it should generate among its students, where the professional has the ability to provide viable solutions to problems of a social nature based on current reality.
\end{abstract}

Keywords: University social responsibility, students, teachers, ethics, management, impact.

Información del manuscrito:

Fecha de recepción: 05 de mayo de 2020.

Fecha de aceptación: 09 de julio de 2020.

Fecha de publicación: 10 de julio de 2020 


\section{Introducción}

Como parte del proceso de formación del estudiante universitario, está el área humanística que los convierte en beneficios para la sociedad, muy independiente de la carrera que cada uno de ellos escoja, dándole a la universidad la potestad de entregar ciudadanos transformados en profesionales reflexivos, con criterio propio hacia el medio que lo rodea, su entorno, la naturaleza con capacidad para ser parte de las transformaciones que sean necesarias para lograr el bien común atendiendo las necesidades colectivas.

Es a partir de esta formación que surge el concepto de responsabilidad social universitaria, donde la UNESCO hace valiosas aportaciones, específicamente con el desarrollo de las últimas conferencias mundiales de Educación Superior llevadas a cabo en 1998 y 2008, donde se destaca que las instituciones de tercer nivel "asuman una mayor responsabilidad social en el contexto del nuevo orden social, sustentado en el acceso a la información y al conocimiento útil y pertinente para atender las problemáticas y necesidades sociales más importantes". (Gaete, 2016)

Es por ello por lo que, la responsabilidad social en el campo universitario no es un fenómeno ajeno a la realidad actual, sino todo lo contrario, es el proceso de construcción donde el estudiante se prepara para laborar en instituciones $\mathrm{u}$ organizaciones públicas y privadas, promoviendo democráticamente los derechos humanos, la ética y una economía más sustentable con el ambiente, donde se sientan responsables de lo que les corresponde como profesionales.

En este sentido (Cardona, 2009), señala que la responsabilidad social es "la capacidad existente en todo sujeto para reconocer y aceptar las consecuencias de un hecho realizado libremente", el cual, en las últimas décadas ha cobrado una gran significancia, dentro de la agenda de las organizaciones públicas y privadas; (Londoño, 2013) refiere que, la responsabilidad social no es una ley ni una teoría, pues no existe un concepto universal que la sustente, ya que implica ir más allá de lo legal e incorporar acciones 
socialmente responsables desde el capital humano, el entorno y las relaciones con los grupos de interés internos y externos.

En este contexto, existe una gran demanda hacia el nivel de educación superior de cuarto nivel, donde se exige que la universidad brinde transferencia de conocimiento direccionado a la sociedad, donde se hace necesaria la gestión organizacional que implique la responsabilidad social, y que para ello hay que sensibilizar y concienciar a los estudiantes como pertenecientes a una sociedad global, con una consciencia de interdependencia porque con ella se puede fortalecer la colaboración en el presente en pro de un futuro mejor; donde la participación ciudadana debe ser enseñada desde el punto de vista colectivo, moral y ético, siendo ésta la base de la responsabilidad social universitaria. (Alférez, 2014)

La Universidad Técnica de Manabí, con su Instituto de Postgrado, no puede quedarse alejada de la reflexión sobre Responsabilidad Social, cuya misión es contribuir al desarrollo de la investigación científica a través de la generación de proyectos que apoyen los programas de formación de los estudiantes de grado y posgrados, así como la vinculación con el sector público y privado, para impulsar la generación del conocimiento y desarrollo de la comunidad científica de la provincia, región y el país.

\section{Desarrollo}

Para analizar el impacto de la responsabilidad social universitaria a nivel mundial se podría decir que ésta empezó desde la Primera Cumbre de la Tierra (1972), en la que se marcó un punto de inflexión en el desarrollo de la política internacional del medio ambiente y se comenzó a hablar de la responsabilidad social. Luego, las Conferencias de la Organización de las Naciones Unidas, sobre el Medio ambiente y el Desarrollo (1992) donde se tomó en consideración el concepto de desarrollo capaz de satisfacer las necesidades actuales sin comprometer los recursos para las próximas generaciones.

Para esto, la Declaración de Talloires (1990) que fue firmada por rectores de más de cuatrocientas universidades del mundo, junto con 
la Declaración Mundial sobre la Educación Superior en el Siglo XXI, la UNESCO (1998), exponen en el 2005-2014, como la era de educación para el Desarrollo Sustentable, señalando que: Desde la perspectiva de la comunidad universitaria estas tendencias le asignan una responsabilidad cada vez mayor en materia de formación, investigación, estudio, asesoría, servicios de orientación, transferencia de tecnología y educación permanente. (UNESCO, 1998)

Para esto en el año 2015 se llevó a cabo la cumbre del desarrollo sostenible, en el cual se destaca el rol de la universidad para fomentar la sostenibilidad, específicamente a partir de la Declaración de Río (1992), la educación universitaria y los centros de educación superior comenzaron a ser visto como un acto clave para proveer soluciones al desarrollo sostenible y en equilibrio con el entorno, ya que son centros de formación de personas y profesionales para el mundo.

En este escenario de cambio continuo, este organismo identifica a la responsabilidad social universitaria como la libertad académica como fuentes indispensables de trabajos esenciales para las instituciones de tercer nivel que logren hacer frente a las nuevas responsabilidades que la sociedad exige a los profesionales.

En América Latina y el Caribe en el año 2000, fue conceptualizada la responsabilidad social universitaria e impulsada principalmente por la Red latinoamericana de universidades, denominada "iniciativa interamericana de ética, capital social y desarrollo", promovida por el Banco Interamericano de Desarrollo de Noruega, el cual estuvo basado en la gestión justa y sostenible de los impactos universitarios, derivando cuatro impactos relacionados directamente con el campo organizacional como son internos y externos (laborales - ambientales), y en el campo académico están los educativos (formación estudiantil) e impactos cognitivos (epistemología e investigación). (Vallaeys \& Carrizo, 2016)

$Y$ es así como a medida que transcurre el tiempo cada vez más las universidades se ajustan a estos enfoques y fortalecen la responsabilidad social, sobre todo por las ventajas que ésta conlleva, 
entre ellas está acentuar el concepto de la responsabilidad social tal como lo define la ISO 26000 "la responsabilidad social de una organización que se responsabiliza de los impactos de la organización hacia la sociedad y el medioambiente", donde la universidad es la encargada de las competencias académicas para su total aplicación por parte del profesional. (Vallaeys, 2014)

Por otra parte, la responsabilidad social universitaria para autores como (De la Cruz \& Sasa, 2008; Gaete, 2011; Licha, 2012 y Vallaeys, 2014), consideran dos impactos de gran relevancia, como es el cognitivo, donde la universidad orienta la producción del conocimiento en ciencia, racionalidad, legitimidad, utilidad, enseñanza, logrando incentivar la separación de los saberes al delimitar los ámbitos de cada carrera; articulando la relación entre tecno ciencia y sociedad, posibilitando el control y la apropiación social del conocimiento, teniendo presente el tipo de conocimiento que produce, por su pertinencia social y por sus destinatarios; en lo referente al impacto social, las instituciones de tercer nivel logra promover el progreso, crear capital social, vincular a los estudiantes con la realidad exterior, hacer accesible el conocimiento, donde su principal objetivo es acompañar el desarrollo de la sociedad y ayudar a resolver sus problemas fundamentales.

En base a lo expuesto, se puede definir que la responsabilidad social contempla varias perspectivas, entre ellas están:

Gerencial: Es una política de mejora continua de la universidad hacia el cumplimiento efectivo de su misión social mediante cuatro procesos: gestión ética y ambiental de la institución, formación de ciudadanos responsables y solidarios, producción y difusión de conocimientos socialmente pertinentes y participación social en promoción de un desarrollo más humano y sostenible (Vallaeys F. , 2014)

Transformacional: Es la habilidad y efectividad de la universidad para responder a este tipo de necesidad, que está inmersa en el ejercicio de sus 
funciones sustantivas como la docencia, la investigación, la extensión y la gestión interna. Para que estas funciones tengan la efectividad deseada, deben estar encaminadas a la búsqueda de la promoción de la justicia, la solidaridad y la equidad social, mediante la construcción de respuestas exitosas para atender los retos que implica promover el desarrollo humano sustentable. (Ausjal, 2015)

Normativo: La capacidad que tiene la universidad como institución, de difundir y poner en práctica un conjunto de principios y valores generales y específicos por medio de los procesos clave de gestión, docencia, investigación y extensión respondiendo socialmente así ante la comunidad universitaria y el país en el que está inserta. (Jiménez, 2014)

Desde un enfoque ético, la responsabilidad social crea un vínculo directo entre la universidad y la sociedad y sus diferentes componentes, donde se pone de manifiesto el compromiso moral que se genera, creando nuevos conocimientos que serán aplicados por el profesional en la búsqueda de soluciones a los diversos problemas sociales, desde un ámbito científico y tecnológico, donde la parte humanitaria es parte importante del quehacer profesional que cumple con la responsabilidad social que como ser humano le corresponde.

En Ecuador, la universidad es vista como un actor trascendental en la sociedad, ya que tiene como principal propósito no solo educar, sino que además transformar con el paso del tiempo a estudiantes en profesionales mediante la generación y transmisión de conocimientos con miras al desarrollo y crecimiento de todo el país, la cual debe ser impartida con una visión humanista, con ética y sobre todo comunitaria, generando de esta forma un impacto positivo ante la sociedad, individual y colectivo. (Espinoza \& Guachamín, 2015)

Un estudio realizado por la revista Internacional de Administración en Ecuador, señala que una de las principales limitaciones que presenta la responsabilidad social en las universidades ecuatorianas, es que no existen datos reales que afirmen la verdadera labor que estas 
instituciones de tercer y cuarto nivel brindan a sus estudiantes y por ende a la sociedad en general.

Para esto, (Mollis, 2010), toma como referencia uno de los principales problemas que representa la educación superior en América Latina el cual señala: "al reconocimiento de la desnaturalización de la misión universitaria", el cual tendría que representar la misión de toda institución educativa, aportar de manera significativa al desarrollo integral de todos los estudiantes, cuando éstos tengan que ejercer cargos públicos y privados, llevando por delante la responsabilidad social como parte de su profesión.

La problemática señalada no se encuentra distante de la realidad de las universidades ecuatorianas, por ello la misión de la educación superior debe conectar de manera directa la interacción universitaria con la responsabilidad social, para que al momento de tomar decisiones éstas estén orientadas a la necesidad de la universidad misma, del contexto donde se ubica, del entender $\mathrm{y}$ atender el quehacer universitario, definiendo desde el primer instante el tipo de papel que juega la responsabilidad social en los campus universitarios. (Soto, 2018)

En base a lo expuesto la universidad y la responsabilidad social merma como una gran oportunidad para poner por delante el papel que desempeña la universidad desde el punto de vista de la sociedad inmersa en todos sus problemas, necesidades, potencialidades, objetivos, metas, entre otros.

El debate sobre la Responsabilidad Social y Universidad surge como una gran oportunidad para repensar el rol de la Universidad desde la perspectiva de nuestras sociedades, sus problemas, sus potencialidades y sus sueños; y donde la programación u organización de la maya curricular universitaria está involucrada de manera directa con el desarrollo y formación del estudiante, con miras a desarrollar la capacidad de atender dichas problemática desde un enfoque social, ético, individual y colectivo, donde todos incluyendo el medio ambiente sean beneficiarios. (Rodríguez et al, 2018)

La Universidad Técnica de Manabí, del cantón Portoviejo, tiene como 
objetivo principal formar integralmente al ser humano para que contribuya al desarrollo del país, al logro de la justicia social, fortaleciendo la identidad nacional en el contexto pluricultural del país, la afirmación de la democracia, la paz, los derechos de las personas y las comunidades, la integración latinoamericana y mundial, así como la defensa y protección del medio ambiente, además de formar, capacitar, especializar y actualizar a sus estudiantes de niveles de pregrado y posgrado, con pensamiento crítico y conciencia social, de manera que contribuyan eficazmente al mejoramiento de la producción intelectual y de bienes y servicios, de acuerdo con las necesidades presente y futuras de la sociedad y los requerimientos del desarrollo nacional; además de brindar una formación científica y humanista, respetuosa de los derechos humanos, la equidad de género y el medio ambiente, que permita a los estudiantes contribuir al desarrollo del país. (UTM, 2020)

En base a lo expuesto, se requiere desarrollar una política de gestión de la calidad ética del Instituto de Postgrado de la Universidad Técnica de Manabí para buscar alinear sus cuatro procesos (gestión, docencia, investigación, extensión) con la misión universitaria, sus valores y compromiso social, mediante el logro de la congruencia institucional, la transparencia y la participación dialógica de toda la comunidad universitaria (autoridades, estudiantes, docentes, administrativos con los múltiples actores sociales interesados en el buen desempeño universitario y necesitados de él, para la transformación efectiva de la sociedad hacia la solución de sus problemas de exclusión, inequidad y sostenibilidad.

\section{Impacto de la responsabilidad social}

De acuerdo con el Reglamento de Carrera y Escalafón del Profesor Investigador del Sistema de Educación Superior (2012) y la Ley Orgánica de Educación Superior (2010), entre las principales actividades que las universidades deben cumplir en unión con el profesorado son: la docencia, la investigación, vinculación con la sociedad y gestión académica, donde la responsabilidad social universitaria debe estar involucrada 
de manera directa en la formación del estudiante.

Para ello (Remolina, 2017), considera que las universidades sean estas públicos o privadas, deben brindar conocimientos profesionales con criterios éticos y humanísticos, de solidaridad y excelencia en el servicio, abarcando puntos importantes como la formación, investigación y extensión. En este sentido (Aguirre et al, 2015) señala que las universidades deben generar la responsabilidad social como compromiso institucional, no como un indicador enmarcado en los principios políticos gubernamentales, lo cual es derivado de la acción y actitud que el profesional tenga con las personas que considera deben recibir su ayuda o colaboración, que los direccione a confirmar el desarrollo de la competitividad en hechos valorativos, significativos, que les den la oportunidad de realizar intercambio de ideas, analizando los comportamientos individuales y colectivos, que la integración sea basada en valores, ética y moral; y que además le permita al profesional empoderarse de las necesidades sociales.
Resulta importante tener presente que en la actualidad la educación superior debe disponer de docentes altamente capacitados, con suficiente capacidad para brindar 0 proporcionar competencias sólidas para enfrentar el mundo de hoy y el del mañana, sino que además está en la obligación de contribuir en la formación de ciudadanos dotados de principios éticos, donde sean ellos los profesionales quienes se sientan comprometidos a ser parte de la construcción de la paz mundial, regional y local, de defender los derechos humanos, los valores humanos y democráticos que le permitan ser entes positivos para la sociedad. (Gaete Q. R., 2016)

Dentro de los impactos que generan la responsabilidad social universitaria están los institucionales y sociales, las cuales deben ser tomadas en cuenta de forma transparente ante las demandas sociales, especialmente cuando las necesidades sociales provengan de grupos de personas vulnerables incapaces de hacerlas entender 0 atender; por ello es imperiosos que todas las acciones que genera la universidad ante el profesional se traduzcan en impactos positivos, tal 
como lo señala (Valleys, 2014) al mencionar que todas las acciones que se ejecuten como parte de la responsabilidad social universitaria tendrán un impacto positivo o negativo ante la gestión universitaria. Destacando los siguientes impactos:

- Académico, estudiantil, administrativo y obrero

- Investigación, generación de saberes y desarrollo de tecnología

- La universidad como institución forma parte del estado, que lo convierte en un ejecutor comprometido con la promoción de acciones encaminadas al bien común y el progreso.

- Finalmente orienta al profesional sin importar la carrera que éste tenga, a la comprensión e interpretación del mundo en sus diversos aspectos, influyendo de manera directa en el desarrollo de valores relacionándola con la ética profesional.

En base a lo expuesto, la UNESCO, señala que se de gran relevancia que toda la comunidad de educación superior participe en la búsqueda de posibles soluciones a ciertos tipos de problemas de carácter humano por citar alguno están la demografía, la paz, el entendimiento internacional, el medioambiente, la democracia, que servirán para comprender con mayor claridad cuáles son verdaderos impactos que generan la responsabilidad social universitaria, pudiendo así recuperar el valor transformador de la Universidad y su papel en la definición y resoluciones de los problemas institucionales como sociales. (UNESCO, 19982015)

Por ello se pone de manifiesto la evaluación como base legal de la responsabilidad Social Universitaria en el Ecuador, según el Mandato Constituyente No. 14, el cual fue decretado el 22 de julio de 2008 la Asamblea Nacional Constituyente dictó el Mandato Constituyente No. 14 cuya Primera Disposición Transitoria dispone que:

"El Consejo Nacional de Educación Superior-CONESUP obligatoriamente en el plazo de un año, deberá determinar la situación académica y jurídica de todas las entidades educativas bajo su control en base al cumplimiento de sus disposiciones y de las normas que sobre educación superior se encuentren vigentes en el país... 
Será obligación que en el mismo período (un año) el Consejo Nacional de Evaluación y Acreditación -CONEA- entregue al CONESUP y a la Función Legislativa, un informe técnico sobre el nivel de desempeño institucional de los establecimientos de educación superior, a fin de garantizar su calidad, propiciando su depuración y mejoramiento, según el artículo 91 de la Ley Orgánica de Educación Superior". (CEAACES, 2013)

El reto de la Responsabilidad Social Universitaria se plantea actualmente en medio de grandes cambios sociales, económicos, políticos y culturales, demandando importantes transformaciones institucionales y cambios en los planteamientos de políticas que impulsen la construcción de nuevos modelos.

\section{Propuesta}

Se propone un modelo para implementar la Responsabilidad Social Universitaria RSU, a partir de un breve análisis de la situación del Instituto de Postgrado de la Universidad Técnica de Manabí, se aplicó la investigación cualitativa, específicamente descriptivaexplicativa, utilizando el ciclo Shewhart (planifique, haga, chequee y actúe). El modelo propuesto parte de los cuatro pilares de la educación superior: docencia, proyección social, investigación y gestión, sumando medio ambiente y posconflicto. El medio ambiente, pese a formar parte del esquema de la responsabilidad social, apenas está siendo tratado con cierto rigor en la literatura, mientras que el último aspecto es de gran relevancia dada la realidad actual del país.

Un potencializado diseño de sistema de gestión de responsabilidad social universitaria dentro del Instituto de postgrado de la Universidad Técnica de Manabí contribuirá al manejo eficiente de la organización y además de formar a los futuros profesionales que laborarán en las empresas, a los futuros ciudadanos que tendrán que promover democráticamente los derechos humanos, y a los futuros funcionarios que tendrán a su cargo el bien común en nuestro mundo globalizado. 


\section{Conclusiones}

La responsabilidad social universitaria es la gestión directa que debería de cumplir todas las universidades sean estas públicas o privadas, responsables de los impactos que generen ante la sociedad.

La responsabilidad social universitaria debe ser evaluada, implementada e investigada por toda la comunidad educativa, incluida en esta estudiantes, docentes, área administrativa e investigadores del área científica, donde los resultados que se obtengan sirvan para incorporar a sus acciones diarias un nuevo modelo de concepción social, perfeccionando todos los esfuerzos y estudios que se realicen por el bienestar no solo de la universidad sino también del futuro profesional en beneficio de la sociedad en general.

En cuanto a la universidad ecuatoriana, la responsabilidad social tiene gran relevancia, $\mathrm{y}$, por tanto, debe ser incorporado procurando la participación de toda la comunidad universitaria, tomando en cuenta que este tema aún está en etapa de formación.
Finalmente, se puede concluir indicando que las universidades de manera general fueron creadas para formar profesionales que puedan responder de manera positiva ante las adversidades, con soluciones a los problemas principalmente de carácter social, considerando que las universidades brindan un escenario donde se fortalecen los talentos humanos y junto con ello fomentar y potencializar los valores éticos y morales.

\section{Bibliografía}

Aguirre, R., Palekais, C., \& Paz, A. (2015). Responsabilidad social: compromiso u obligación universitaria. Telos. Revista de estudios interdisciplinarios en ciencias sociales, 14(1), 11-20.

Alférez, V. A. (2014). Responsabilidad Social Universitaria en la formaciónm curricular y la participación social: El caso de la Facultad de Educación de la universitat Internacional de Catalunya. Catalunya: Universitat Internacional de Catalunya.

Ausjal. (1 de Octubre de 2015). Presentación sintetizada del sistema de autoevaluación y gestión de la responsabilidd social universitaria en AUSJAL. Obtenido de 
http://www.ausjal.org/tl_files/a usjal/

images/contenido/Documento s/

Publicaciones/Documentos\% 20

Institucionales/RSU\%20AUS

JAL\%20

version\%20acotada.pdf

Cardona, A. (2009). Una aproximación sobre lo que es la responsabilidad social empresarila. Saber ciencia libertad. Revista del Centro de Investigaciones de la Universidad Libre Cartagena, 161-167.

CEAACES. (5 de Mayo de 2013). Informe de gestión. Obtenido de

http://gob.ec/images/stories/d ocumentacion/mandato_14/in forme_2009/3_uni ersidades/Inf_univ_1.pdf

De la Cruz, C., \& Sasia, P. (2008). La responsabilidad de la universidad en el proyecto de construcción de una sociedad. Revista Educación Superior y Sociedad, 13(2); 17-52.

Espinoza, S. G., \& Guachamín, M. M. (2015). La responsabilidad social universitaria en Ecuador. Estudios de la Gestión. Revista internacional de administración, 19.

Gaete, Q. R. (Noviembre de 2016). Iniciativas internacionales $y$ redes interuniversitarias de responsabilidad social universitaria. Redalyc, Ciencia, Docencia y Tecnología, 27(53), 75-102. Obtenido de https://www.redalyc.org/pdf/1 45/14548520004.pdf

Gaete, R. (2011). La responsabilidad social universitaria como desafío para la gestión estratégica de la Educación Superior: el caso de España. Revista de Educación, 109133.

Jiménez, M. (25 de Septiembre de 2014). Educar para la responsabilidad social. Santiago de Chile. Obtenido de http://www.spring-alfapucv. cl/wpcontent/uploads/2013/03/Edu car-para-la-responsabilidadsocial-universitaria-MonicaJimenez.pdf

Licha, I. (2012). La responsabilidad social de la educación superior. Enseñanza de la responsabilidad social empresarial retos de las universidades en Iberoamérica. Buenos Aires: PNUD.

Londoño, F. I. (2013). Responsabilidad Social Universitaria. Una estrategia de gestión para la educación superior. Armenia: Sinapsis. Escuela de Administración y Mercadotecnica de Quindio. 
Mollis, M. (2010). La transformación de la educación superior en América Latina: Identidades de construcción. . Ecuador: UNESCO - IESALC. Educación Superior y Sociedad.

Remolina, G. (2017). La responsabilidad social de la universidad. Nómadas. № 19: 239-746.

Rodríguez, A. G., Cano, L. E., \& Vélez, R. X. $(26$ de Septiembre de 2018). Responsabilidad social universitaria: un enfoque a la relación de la universidad pública con el estudiante. Obtenido de Doi: https://doi.org/10.33936/eca_ sinergia.v9i1.962

Soto, D. O. (2018). La responsabilidad social en la universidad ecuatoriana. Revista electrónica en Iberoamérica especializada en comunicación. Razón y Palabra, 20.

UNESCO. (1998). Autonomía, responsabilidad socia y libertad académica, VII Conferencia Mundial de Educación. Recuperado el 1 de Octubre de 2015, de http://unesdoc.unesco.org/im ages/0011/001135/113549so. pdf

UNESCO. (1998-2015). La educación superior en el siglo XXI. Visión y acción.
Documento de trabajo. París. (ED-98/CONF. 202/5: Conferencia mundial sobre la educación superior.

UTM. (6 de Junio de 2020). Historia, objetivos, metas y propósitos de la Universidad Técnica de Manabí . Obtenido de https://www.utm.edu.ec/launiversidad/quienes-somos

Vallaeys, F. (2014). La responsabilidad social universitaria: un nuevo modelo universitario contra la mercantilización. Scielo. Revista iberoamericana de educación superior, 5(12). Obtenido de http://www.scielo.org.mx/sciel o.php?script=sci_arttext\&pid= S2007-28722014000100006

Vallaeys, F., \& Carrizo, L. (2016). Responsabilidad social universitaria: marco conceptual, antecedentes, herramientas. Lima - Perú: CD Multimedia, Banco Interameericano de Desarrollo. 